\title{
CONVEXITY OF THE EXERCISE BOUNDARY OF THE AMERICAN PUT OPTION ON A ZERO DIVIDEND ASSET
}

\author{
Xinfu Chen AND John Chadam \\ University of Pittsburgh \\ LISHANG JIANG \\ Tongji University \\ WEIAN ZHENG \\ University of California, Irvine
}

\begin{abstract}
We show that the optimal exercise boundary for the American put option with non-dividend-paying asset is convex. With this convexity result, we then give a simple rigorous argument providing an accurate asymptotic behavior for the exercise boundary near expiry.
\end{abstract}

KEY WORDS: American put option on a zero dividend asset, convexity of the early exercise boundary, free boundary problem, obstacle problem, near-expiry estimates.

\section{INTRODUCTION}

The Black-Scholes model is widely used to value options. An important advantage of the model is that European options can be valued analytically by the Black-Scholes formula (Merton 1992; Hull 1997). The situation is quite different, however, for American put options with optimal early exercise. While considerable progress has been made, no completely satisfactory analytic solution has been found. As a result, people resort routinely either to numerical methods or to analytic approximations. There is a considerable literature in these fields; see, for example, McKean (1965), Van Moerbeke (1976), MacMillan (1986), Barone-Adesi and Whaley (1987), Barone-Adesi and Elliott (1991), Carr (1992), Barle (1995), Broadie and Detemple (1996), Hull (1997), Kuske and Keller (1998), and Stamicar (1999). A recent list of references can be found in Aitsahlia and Lai (2001) and Chen and Chadam (2006). With the hypothesis of log-normal underlying asset pricing $S$ and based on standard arbitrage-free arguments, the price $P(S, T)$ (T-current time) for the American put option on a non-dividend-paying asset can be formulated as an

Chadam and Chen thank the National Science Foundation for financial support of this research, the first from grant DMS-9704567 and the second from grants DMS-9971043, 0203991, and 0504691. Jiang thanks the National Natural Science Foundation of China for financial support of this research from grant no. 19871062. The authors would like to thank the reviewers and editors for their careful reading of the manuscript and for several helpful comments.

Manuscript received July 2001; final revision received August 2006.

Address correspondence to John Chadam, Department of Mathematics, Thackeray Hall 512, University of Pittsburgh, Pittsburgh, PA 15260, USA; e-mail: chadam@pitt.edu. 
obstacle problem (Wilmott 1995). The domain $\left\{0 \leq T \leq T_{F}, 0 \leq S<\infty\right\}\left(T_{F}\right.$-expiration time) is separated by the optimal exercise boundary $S=S_{f}(T)$ into two parts:

(i) a continuation region $\left\{S_{f}(T)<S<\infty, 0 \leq T \leq T_{F}\right\}$ where $P(S, T)>$ Payoff $=$ $(\mathrm{E}-\mathrm{S})^{+}(=: \max \{0, E-S\})$ and satisfies the Black-Scholes equation

$$
\frac{\partial P}{\partial T}+\frac{1}{2} \sigma^{2} S^{2} \frac{\partial^{2} P}{\partial S^{2}}+r S \frac{\partial P}{\partial S}-r P=0 ;
$$

(ii) a stopping region $\left\{0 \leq S \leq S_{f}(T), 0 \leq T \leq T_{F}\right\}$ where $P(S, T)=$ Payoff $=$ $(\mathrm{E}-\mathrm{S})^{+}$.

Across the optimal exercise boundary $S=S_{f}(T), P$ and $\frac{\partial P}{\partial S}$ are continuous. Here $E$ is the exercise (strike) price, $\sigma$ the volatility constant, and $r$ the risk-free interest rate.

By using a PDE argument, it is not difficult to show that

$$
S_{f}(T) \text { is a monotonically increasing function of } T \text { and } S_{f}\left(T_{F}\right)=E \text {. }
$$

Thus, in the continuation region, the price $P(S, T)$ of the American put option is a solution to the following free boundary problem:

$$
(P) \begin{cases}P_{T}+\frac{1}{2} \sigma^{2} S^{2} P_{S S}+r S P_{S}-r P=0 & \text { for } 0 \leq T<T_{F}, S>S_{f}(T), \\ P(S, T)=E-S, P_{S}(S, T)=-1 & \text { for } 0 \leq T<T_{F}, S=S_{f}(T), \\ P\left(S, T_{F}\right)=(E-S)^{+}, S_{f}\left(T_{F}\right)=E & \text { for } T=T_{F}, S \geq 0 .\end{cases}
$$

For notational simplicity, it is convenient to write $(\mathrm{P})$ in a non-dimensional form. Let

$$
k=2 r / \sigma^{2}, \quad S=E e^{x}, \quad T=T_{F}-2 t / \sigma^{2}, \quad P(S, T)=E p(x, t), \quad S_{F}(T)=E e^{s(t)} .
$$

Then the problem $(\mathrm{P})$ becomes, for the transformed price $p(x, t)$ and exercise boundary $x=s(t)$,

$$
\begin{cases}p_{t}-p_{x x}-(k-1) p_{x}+k p=0 & \text { for } t>0, x>s(t), \\ p(s(t), t)=1-e^{s(t)}, p_{x}(s(t), t)=-e^{s(t)} & \text { for } t>0, x=s(t), \\ p(x, 0)=\left(1-e^{x}\right)^{+} & \text {for } t=0,0 \leq x<\infty .\end{cases}
$$

Here we have $p(x, t) \equiv 1-e^{x}$ all for $x<s(t)$ and

$$
s(0)=0, \quad \dot{s}(t):=\frac{d}{d t} s(t)<0 \text { for all } t>0 .
$$

In this paper, we shall show that the exercise boundary is convex; namely, we prove the following theorem:

THEOREM 1.1. The optimal exercise boundary $S_{f}(T)$ is convex and the transformed boundary $s=s(t)$ is convex; namely,

$$
\frac{d^{2}}{d T^{2}} S_{f}(T)>0 \text { for all } T<T_{F}, \quad \ddot{s}(t)>0 \text { for all } t>0 .
$$

Since $\frac{d^{2}}{d T^{2}} S_{f}(T)=\sigma^{4} E e^{s(t)}\left\{\ddot{s}(t)+\dot{s}^{2}(t)\right\} / 4$, we see that $\ddot{s}(t)>0$ implies $\frac{d^{2}}{d T^{2}} S_{f}(T)>0$. We would like to point out that numerical simulations in the past had already convinced people that $S_{f}(T)$ is convex. Nevertheless, prior to our current work, we had not seen any published research on the rigorous verification of this important fact. During the revision of this paper, an alternate rigorous proof of the convexity has appeared (Ekstrom 
2004). Both proofs are based on the use of the classic results of Friedman and Jensen (1997). Ekstrom (2004) uses the level curves of $v=\left(p+e^{x}\right)_{t} /\left(p+e^{x}\right)_{x}$ while our analysis is based on studying $\phi=q_{t} / q_{x}$ where $q$ satisfies the classic Stefan problem with deltafunction initial data and is related to $p$ in equation (1.1) through $p(x, t)=\left(1-e^{x}\right)^{+}+$ $\int_{0}^{t} q(x, \tau), d \tau$ and hence, $p_{t}=q$. Another interesting difference in the proofs is that Ekstrom (2004) uses a version of the near expiry estimate (1.2) below in his proof to establish a decreasing order for the level curves $v=\alpha$ away from the early exercise boundary. By contrast, one of our main objectives is to prove the convexity in order to provide a simpler proof of these estimates. Specifically, in Section 4, we will use the convexity to prove

THEOREM 1.2. Near expiry, the optimal exercise boundary satisfies

$$
\begin{aligned}
\lim _{t \searrow 0}\left\{\frac{s^{2}(t)}{4 t}+\log \sqrt{4 \pi k^{2} t}\right\} & =0, \\
\log \left[S_{f}(T) / E\right] & =\sigma \sqrt{\left(T_{F}-T\right)\left[-\log \left(8 \pi r^{2}\left(T_{F}-T\right) / \sigma^{2}\right)+o(1)\right]}
\end{aligned}
$$

where $o(1) \rightarrow 0$ ad $T \nearrow T_{F}$.

Note that the behavior of $S_{f}(T)$ follows directly from the behavior of $s(t)$.

There have been many efforts on the near expiry behavior of the exercise boundary (Barone-Adesi and Whaley 1987; Barles et al. 1995; Kuske and Keller 1998; Stamicar et al. 1999; Bunch and Johnson 2000; Chen and Chadam 2006). The correct coefficient $4 \pi k^{2}$ in (1.2) was first found in Stamicar et al. (1999), and later rigorously verified in Chen and Chadam (preprint), with a very involved mathematical analysis. As mentioned above, the argument to be presented here is much simpler. The near expiry behavior and convexity of the optimal exercise boundary can then be interpolated with the long time behavior (the infinite horizon solution) to provide a global, in time, approximation for the exercise boundary; see, for example, Bunch and Johnson (2000), Chen and Chadam (2006).

It would be interesting to examine convexity for problems with other pay-offs and/or underliers. Indeed with the same approach we find that the optimal boundary $S_{f}(T)$ (not necessarily $s(t))$ is convex when the payoff $(E-S)^{+}$is replaced by certain other functions whose derivative admits a jump at $S=E$. On the other hand, we anticipate that a full study of these questions might be quite subtle and specialized in view of the fact that numerical simulations for the closely related problem (P) on a dividend-paying asset suggest that for a particular choice of parameters, the early exercise boundary may not be convex ( $\mathrm{J}$. Detemple, private communication).

In this paper we will show $\ddot{s}>0$ by studying the equation for $q(x, t)=p_{t}(x, t)$ which satisfies

$$
\begin{cases}q_{t}-\mathbf{L} q=0 & \text { for } x>s(t), t>0, \\ q=0, q_{x}=-k \dot{s} & \text { on } x=s(t), t>0, \\ q(x, 0)=\delta(x) & \text { for } x \geq 0,\end{cases}
$$

where $\delta(x)$ is the Dirac function with mass concentrated at the right-half of the origin, ${ }^{*}=$ $\frac{d}{d t}$ and $\boldsymbol{L}$ represents the operator

$$
\mathbf{L} q=\left\{\frac{\partial^{2}}{\partial x^{2}}+(k-1) \frac{\partial}{\partial x}-k\right\} q .
$$


Formally the system (1.3) satisfied by $q$ can be obtained as follows (a rigorous proof will be given in Section 3). First of all, (1.3) can be derived by differentiating equation (1.1) with respect to $t$. The equation $q_{t}=\boldsymbol{L} q$ follows by differentiating $p_{t}=\mathbf{L} p$ with respect to $t$. The boundary condition $q=0$ on $x=s(t)$ follows from the fact that $q=p_{t}$ is continuous in $\mathbb{R} \times(0, \infty)$. The free boundary condition $q_{t}=k \dot{s}$ on $x=s(t)$ follows from

$$
\begin{aligned}
q_{x}(s(t)+, t)=p_{x t} & =\frac{d}{d t} p_{x}-p_{x x} \dot{s}=\left[-e^{s}-p_{x x}\right] \dot{s} \\
& =\left[-e^{s}-p_{t}+(k-1) p_{x}-k p\right] \dot{s}=-k \dot{s} .
\end{aligned}
$$

Finally, $q(x, 0)=\mathbf{L}\left(1-e^{x}\right)^{+}=\delta(x)-k \chi_{\{x<0\}}$ giving the initial condition in (1.3) in the region $x \geq 0$. The proof then employs the idea that for a solution $q$ to (1.3), the function

$$
p(x, t):=\left(1-e^{x}\right)^{+}+\int_{0}^{t} q(x, \tau) d \tau
$$

solves equation (1.1), so that $p_{t}=q$. Note that the first part $\left(1-e^{x}\right)^{+}$represents the exercise pay-off of the put option. Thus the second quantity is the delayed exercise premium and the function $q(x, \tau)$ represents the local benefits of delaying exercise at $\tau$; see Carr, Jarrow, and Myneni (1997). Since $s(\cdot)$ is decreasing and $q(x, \tau)=0$ for all $x<s(\tau)$, we see that $p(x, t)=\left(1-e^{x}\right)^{+}$for all $x \leq s(t)$. Thus all information about the exercise boundary is captured in problem (1.3).

One notices that problem (1.3) is a one-phase Stefan problem with Dirac function as its initial value. The influence of initial datum on the curvature of the free boundary $s(t)$ to one-phase Stefan problem for the heat equation has been studied by Friedman and Jensen (1977). The major tool is to study the level curves of the function $\phi(x, t)=\frac{q_{t}(x, t)}{q_{x}(x, t)}$ where $q$ is a solution to the one-phase Stefan problem. But this approach will not carry over directly for the problem (1.3) since here the initial datum is the singular Dirac function. We shall approximate (1.3) with special smooth initial data and prove $\ddot{s}>0$ for the approximation problems by using the idea introduced in Friedman and Jensen (1977). Then we take the limit to show the convexity of the exercise boundary.

We remark that the approximation problem we present can also be used for numerical simulations. This approximation handles the singularity near the origin nicely, and hence could improve numerical accuracy and speed.

Our scheme is as follows. We first replace the initial data for (1.3) by a sequence $\left\{q_{0}^{\varepsilon}(x)\right\}$ of smooth functions approximating the Dirac mass. For the resulting solution $\left(q^{\varepsilon}, s^{\varepsilon}\right)$, we define $p^{\varepsilon}=p_{0}^{\varepsilon}(x)+\int_{0}^{t} q^{\varepsilon}(x, \tau) d \tau$ where $p_{0}^{\varepsilon}$ is the unique solution of $\mathbf{L} p_{0}^{\varepsilon}=$ $q_{0}^{\varepsilon}(x) \chi_{\{x>0\}}-k \chi_{\{x<0\}}$.

We show that (i) $\left(p^{\varepsilon}, s^{\varepsilon}\right) \rightarrow(p, s)$ uniformly as $\varepsilon \searrow 0$, where $(p, s)$ is the unique solution to equation (1.1), and (ii) with a carefully chosen Dirac mass approximating sequence $\left\{q_{0}^{\varepsilon}\right\}, s^{\varepsilon}$ is convex. As a consequence, $s=\lim s^{\varepsilon}$ is also convex. Since $q^{\varepsilon}=p_{t}^{\varepsilon}$, we see that $\lim q^{\varepsilon}=p_{t}$ and that $q=: p_{t}=\lim q^{\varepsilon}$ is a solution to (1.3) in a certain weak mathematical sense, which we shall not elaborate upon.

\section{THE APPROXIMATION PROBLEM}

We consider, for every $\varepsilon>0$, the following problem, for $\left(q^{\varepsilon}(x, t), s^{\varepsilon}(t)\right)$,

$$
\begin{cases}q_{t}^{\varepsilon}-\mathbf{L} q^{\varepsilon}=0 & \text { for } x>s^{\varepsilon}(t), t>0, \\ q^{\varepsilon}=0, q_{x}^{\varepsilon}=-k \dot{s}^{\varepsilon} & \text { on } x=s^{\varepsilon}(t), t>0, \\ q^{\varepsilon}(x, 0)=q_{0}^{\varepsilon}(x) & \text { for } x \geq s^{\varepsilon}(0):=0 .\end{cases}
$$


Here $q_{0}^{\varepsilon}$ is a non-negative function approximating the Dirac function and will be chosen carefully such that $s^{\varepsilon}(t)$ is convex.

In the sequel, a solution $q^{\varepsilon}$ to (2.1) is automatically extended to the domain $x \in \mathbb{R}, t \geq 0$ by the default

$$
q^{\varepsilon}(x, t) \equiv 0 \quad \forall x \leq s^{\varepsilon}(t), \quad t \geq 0 .
$$

In this section, we first study the well-posedness of (2.1), then construct $p^{\varepsilon}$ from the equation $p_{t}^{\varepsilon}=q^{\varepsilon}$ as in equation (2.4) below, and finally show the convergence of $\left(p^{\varepsilon}, s^{\varepsilon}, q^{\varepsilon}\right)$ to $(p, s, q)$ as $\varepsilon \searrow 0$. As a consequence, we have $q=p_{t}$.

We begin with the well-posedness of (2.1).

LeMma 2.1. Assume that $q_{0}^{\varepsilon}$ satisfies

$$
\left\{\begin{array}{l}
q_{0}^{\varepsilon} \in C^{4}([0, \infty)) \cap L^{1}((0, \infty)), \quad q_{0}^{\varepsilon}(x)>0 \quad \text { for all } x>0, \\
q_{0}^{\varepsilon}(0)=0, \quad q_{0 x}^{\varepsilon}(0)^{2}=k \mathbf{L} q_{0}^{\varepsilon}(0), \quad q_{0}^{\varepsilon}(\infty):=\lim _{x \rightarrow \infty} q_{0}^{\varepsilon}(x)=0 .
\end{array}\right.
$$

Then problem (2.1) admits a unique solution, and the solution satisfies, for any $\alpha \in(0,1)$,

$$
\begin{aligned}
& s^{\varepsilon} \in C^{\infty}((0, \infty)) \cap C^{2+\alpha / 2}([0, \infty)), \quad \dot{s}^{\varepsilon}<0 \text { on }[0, \infty), \\
& q^{\varepsilon} \in C^{\infty}\left(D^{\varepsilon}\right) \cap C^{3+\alpha,(3+\alpha) / 2)}\left(\bar{D}^{\varepsilon}\right), \quad q^{\varepsilon}>0 \text { in } D^{\varepsilon}=\left\{(x, t) \mid x>s^{\varepsilon}(t), t>0\right\} .
\end{aligned}
$$

Since (2.1) is a one-phase Stefan problem, the result is well-known and hence the proof is omitted; see, for example, Jiang (1965) or Friedman (1982, 1976). We remark that the condition on $q_{0}^{\varepsilon}(0)$ and $q_{0 x}^{\varepsilon}(0)$ are simply the zeroth and first order compatibility conditions: the values of $q^{\varepsilon}$ and $q_{t}^{\varepsilon}=\mathbf{L} q^{\varepsilon}$ at the origin $(0,0)$ calculated from the initial data match with that calculated from the free boundary conditions. Indeed, $0=\dot{q}^{\varepsilon}\left(s^{\varepsilon}, t\right)=$ $q_{t}^{\varepsilon}+q_{x}^{\varepsilon} \dot{s}=q_{t}^{\varepsilon}-q_{x}^{\varepsilon 2} / k$. Since $q_{t}^{\varepsilon}=\mathbf{L} q^{\varepsilon}$ at the origin, we need $k \mathbf{L} q_{0}^{\varepsilon}=q_{0 x}^{\varepsilon 2}$ at $x=0$. These compatibility conditions ensure that the PDE in (2.1) extends continuously to the free boundary for $t \geq 0$.

To recover $p$, we first extend the solution $q^{\varepsilon}$ of (2.1) by (2.2) and then define $p^{\varepsilon}$ by

$$
p^{\varepsilon}(x, t):=p_{0}^{\varepsilon}(x)+\int_{0}^{t} q^{\varepsilon}(x, \tau) d \tau, \quad x \in \mathbb{R}, t \geq 0
$$

where $p_{0}^{\varepsilon} \in C^{1}(\mathbb{R}) \cap L^{\infty}(\mathbb{R})$ is the solution to the following ODE problem

$$
\mathbf{L} p_{0}^{\varepsilon}= \begin{cases}q_{0}^{\varepsilon}(x) & \text { if } x>0, \\ -k & \text { if } x<0 .\end{cases}
$$

This definition comes from $q=p_{t}=\mathbf{L} q$, the fact that $p=p_{0}=: \max \left\{1-e^{x}, 0\right\}$ for $x<$ $s(t)$, and the identity $\mathbf{L} p_{0}(x)=-k$ for $x<0$.

One notices that the second order derivative $p_{0 x x}^{\varepsilon}$ has a jump $q_{0}^{\varepsilon}(0)-(-k)=k$ when $x$ passes through 0 .

First we show that $p_{0}^{\varepsilon}$ is well-defined.

Lemma 2.2. Assume that $q_{0}^{\varepsilon}$ satisfies (2.3). Then (2.5) admits a unique solution

$$
p_{0}^{\varepsilon} \in C^{1}(\mathbb{R}) \cap L^{\infty}(\mathbb{R}) \cap C^{\infty}((-\infty, 0-]) \cap C^{6}([0+, \infty)) .
$$


In addition, suppose that as $\varepsilon \rightarrow 0, q_{0}^{\varepsilon} \rightarrow \delta$, i.e.,

$$
1=\lim _{\varepsilon \searrow 0} \int_{0}^{\infty} q_{0}^{\varepsilon}(x) d x=\lim _{\varepsilon \searrow 0} \int_{0}^{z} q_{0}^{\varepsilon}(x) d x \quad \forall z>0 .
$$

Then $\lim _{\varepsilon \searrow 0} p^{\varepsilon}{ }_{0}(x)=p_{0}(x)=: \max \left\{1-e^{x}, 0\right\}$ uniformly for $x \in \mathbb{R}$.

The proof is elementary and is given in the Appendix.

THEOREM 2.1. Assume that $q_{0}^{\varepsilon}$ satisfies (2.3) and (2.6). Let $p_{0}^{\varepsilon}$ be the unique solution to (2.5), $\left(q^{\varepsilon}, s^{\varepsilon}\right)$ be the unique solution to (2.1), and define $p^{\varepsilon}$ as in (2.4). Then

$$
\lim _{\varepsilon \searrow 0} p^{\varepsilon}(x, t)=p(x, t), \quad \lim _{\varepsilon \searrow 0} s^{\varepsilon}(t)=s(t) \text { uniformly for all } x \in \mathbb{R}, t \geq 0
$$

where $(p, s)$ is the unique solution to equation (1.1).

Proof. Since $q^{\varepsilon}$ is continuous, one can verify $p^{\varepsilon} \in C^{1}(\mathbb{R} \times[0, \infty))$. As $q^{\varepsilon}=0$ for $x \leq s^{\varepsilon}(t)$,

$$
p^{\varepsilon}(x, t)-p_{0}^{\varepsilon}(x)=p_{x}^{\varepsilon}(x, t)-p_{0 x}^{\varepsilon}(x)=0 \quad \forall x \leq s^{\varepsilon}(t), t \geq 0 .
$$

When $x>s^{\varepsilon}(t), q^{\varepsilon}>0$, so that $p^{\varepsilon}>p_{0}^{\varepsilon}$. In addition, one calculates

$$
p_{t}^{\varepsilon}-\mathbf{L} p^{\varepsilon}=-\mathbf{L} p_{0}^{\varepsilon}+q_{0}^{\varepsilon}(x) \chi_{\{x>0\}}-k \chi_{\{x<0\}}=0 \quad \forall x>s^{\varepsilon}(t), t \geq 0
$$

by the definition of $p_{0}^{\varepsilon}$. In the set $x<s^{\varepsilon}(t)$, we have $p^{\varepsilon}=p_{0}^{\varepsilon}$ so $p_{t}^{\varepsilon}-\mathbf{L} p=-\mathbf{L} p_{0}^{\varepsilon}=k>0$. Thus, $p^{\varepsilon}$ indeed solves the following obstacle problem

$$
\begin{cases}p_{t}^{\varepsilon}-\mathbf{L} p^{\varepsilon} \geq 0, p^{\varepsilon}-p_{0}^{\varepsilon} \geq 0,\left(p^{\varepsilon}-p_{0}^{\varepsilon}\right)\left(p_{t}^{\varepsilon}-\mathbf{L} p^{\varepsilon}\right)=0 & \text { in } \mathbb{R} \times(0, \infty), \\ p^{\varepsilon}(x, 0)=p_{0}^{\varepsilon}(x) & \text { on } \mathbb{R} \times\{0\}\end{cases}
$$

As $k>0$, a comparison principle for the obstacle problem (see, for example Friedman 1982) gives

$$
\left\|p^{\varepsilon}-p\right\|_{C(\mathbb{R} \times[0, \infty))} \leq\left\|p_{0}^{\varepsilon}-p_{0}\right\|_{C(\mathbb{R})} .
$$

Since $p_{0}^{\varepsilon} \rightarrow p_{0}$ uniformly as $\varepsilon \rightarrow 0$, we see that $p^{\varepsilon} \rightarrow p$ uniformly. In addition, since $p_{t}=q \geq 0$, one can show that the coincidence set $\left\{(x, t) \mid p^{\varepsilon}=p_{0}^{\varepsilon}\right\}=\left\{(x, t) \mid x \leq s^{\varepsilon}(t), t \geq\right.$ $0\}$ also approaches that of the limit problem as $\varepsilon \rightarrow 0$. (Indeed, once we know the regularity of $s$, one can show that $\left.\left(p-p_{0}\right)_{x x}\right|_{s=s^{+}}=k$ for every $t>0$. ) This completes the proof.

In the next section, we show that for an appropriate Dirac sequence $\left\{q_{0}^{\varepsilon}\right\}$, $s^{\varepsilon}$ is convex and that $\left(q^{\varepsilon}, s^{\varepsilon}\right) \rightarrow(q, s)$. As $q^{\varepsilon}=p_{t}^{\varepsilon}$, we see that $p_{t}=q$.

\section{THE CONVEXITY OF $s^{\varepsilon}$}

As mentioned in the introduction, we use the idea of Friedman and Jensen (1977) considering the function $\phi^{\varepsilon}=q_{t}^{\varepsilon} / q_{x}^{\varepsilon}$. The basic idea is to choose an appropriate $\delta$ sequence $\left\{q_{0}^{\varepsilon}\right\}_{0<\varepsilon<\varepsilon_{0}}$ such that the derivatives $q_{x}^{\varepsilon}$ and $q_{t}^{\varepsilon}$ have the signs illustrated in the following figure.

More precisely, (i) the set $\left\{q_{x}^{\varepsilon}=0\right\}$ is a smooth curve $x=s_{1}^{\varepsilon}(t)$, to its left $q_{x}^{\varepsilon}>0$ and to its right $q_{x}^{\varepsilon}<0$; (ii) in the region bounded by $x=s^{\varepsilon}(t)$ and $x=s_{1}^{\varepsilon}(t)$, there is a smooth curve $x=s_{2}^{\varepsilon}(t)$ on which $q_{t}^{\varepsilon}=0$, to its left, $q_{t}^{\varepsilon}<0$ and to its right, $q_{t}^{\varepsilon}<0$. 
First we consider the curve on which $q_{x}^{\varepsilon}=0$.

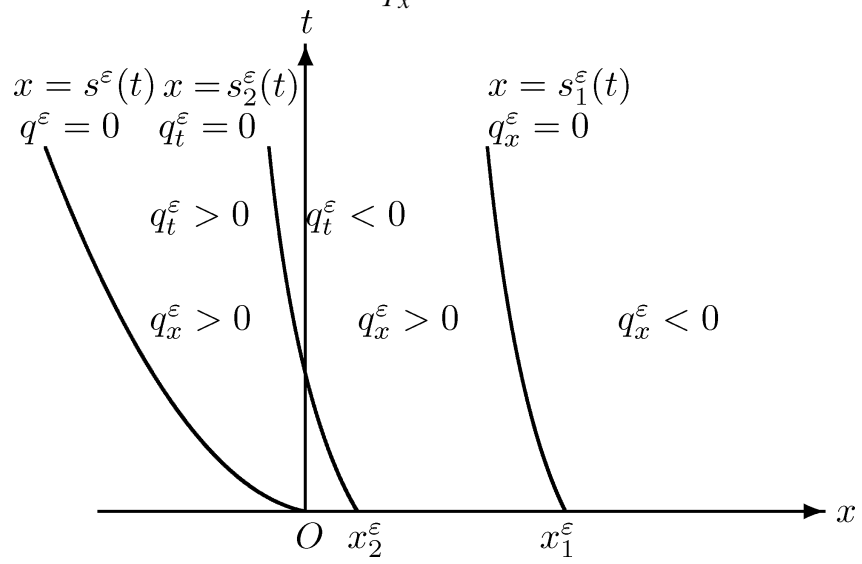

Lemma 3.1. Assume that $q_{0}^{\varepsilon}$ satisfies (2.3), (2.6), and for some $x_{1}^{\varepsilon}>0, q_{0 x}^{\varepsilon}\left(x_{1}^{\varepsilon}\right)=0$ and

$$
q_{0 x}^{\varepsilon}(x)>0 \forall x \in\left[0, x_{1}^{\varepsilon}\right), \quad q_{0 x}^{\varepsilon}(x)<0 \forall x \in\left(x_{1}^{\varepsilon}, \infty\right), \quad q_{0 x x}^{\varepsilon}\left(x_{1}^{\varepsilon}\right)<0 .
$$

Then there exists a smooth function $s_{1}^{\varepsilon}(t)$ defined for $t \in[0, \infty)$ such that $s_{1}^{\varepsilon}(0)=x_{1}^{\varepsilon}$ and for all $t \geq 0$,

$$
\begin{gathered}
s_{1}^{\varepsilon}(t)>s^{\varepsilon}(t), \quad q_{x}^{\varepsilon}\left(s_{1}^{\varepsilon}(t), t\right)=0, \quad q_{x x}^{\varepsilon}\left(s_{1}^{\varepsilon}(t), t\right)<0, \quad q_{t}^{\varepsilon}\left(s_{1}^{\varepsilon}(t), t\right)<0, \\
q_{x}^{\varepsilon}(x, t)>0 \text { if } x \in\left[s^{\varepsilon}(t), s_{1}^{\varepsilon}(t)\right), \quad \text { and } q_{x}^{\varepsilon}(x, t)<0 \text { if } x>s_{\varepsilon}^{1}(t) .
\end{gathered}
$$

Proof. Note that $q^{\varepsilon}>0$ in $D^{\varepsilon}=:\left\{(x, t) \mid x>s^{\varepsilon}(t), t>0\right\}$ and $0=q^{\varepsilon}\left(s^{\varepsilon}(t), t\right)$ for all $t \geq$ 0 . Also, since $q_{0}^{\varepsilon}(\infty)=: \lim _{x \rightarrow \infty} q_{0}^{\varepsilon}(x)=0$ we have $q^{\varepsilon}(\infty, t)=0$ for every $t \geq 0$. Thus there exists a point $s_{1}^{\varepsilon}(t) \in\left(s^{\varepsilon}(t), \infty\right)$ on which $q^{\varepsilon}(\cdot, t)$ attains its positive maximum. Clearly, $q_{x}^{\varepsilon}\left(s_{1}^{\varepsilon}(t), t\right)=0$. Next we show that $s_{1}^{\varepsilon}$ is unique.

Since $q^{\varepsilon}$ attains its minimum at $x=s^{\varepsilon}(t)$, by Hopf's Lemma, $q_{x}^{\varepsilon}\left(s^{\varepsilon}(t), t\right)>0$. From the $\operatorname{PDE}\left(q_{x}^{\varepsilon}\right)_{t}-\mathbf{L} q_{x}^{\varepsilon}=0$ in $\bar{D}^{\varepsilon}$, we conclude that the number of roots, for $z \in\left[s^{\varepsilon}(t), \infty\right)$, to $q_{x}^{\varepsilon}(z, t)=0$ is non-increasing in $t$. Since initially $q_{x}^{\varepsilon}(z, 0)=0$ has exactly one root attained at $x_{1}^{\varepsilon}$, we conclude that $q_{x}^{\varepsilon}(z, t)=0$ has at most one, and hence exactly one root. Thus, $s_{1}^{\varepsilon}$ is uniquely defined. Consequently, $q_{x}^{\varepsilon}>0$ for $x \in\left[s^{\varepsilon}(t), s_{1}^{\varepsilon}(t)\right)$ and $q_{x}^{\varepsilon}<0$ for $x>s_{1}^{\varepsilon}$.

To show that $s_{1}^{\varepsilon}(t)$ is smooth, we need only show that $q_{x x}^{\varepsilon}\left(s_{1}^{\varepsilon}(t), t\right)<0$ since the implicit function theorem then can be applied to the equation $q_{x}^{\varepsilon}(z, t)=0$ for $z$.

To do this, we use Sard's theorem, which asserts that there are arbitrarily small $\delta>0$ such that the set where $q_{x}^{\varepsilon} e^{k t} \pm \delta=0$ are smooth curves. Since $\left(q_{x}^{\varepsilon} e^{k t}\right)_{t}=\left(q_{x}^{\varepsilon} e^{k t}\right)_{x x}+(k-$ 1) $\left(q_{x}^{\varepsilon} e^{k t}\right)_{x}$, one can use the same idea as above to show that the zero level set of $q_{x}^{\varepsilon} e^{k t} \pm \delta$, for $t \geq 0$, is actually a vertical curve (i.e., no up and down swings). Now we consider $q_{x x}^{\varepsilon}$ in the region $\left\{(x, t)|| q_{x}^{\varepsilon} \mid e^{k t} \leq \delta, 0<t \leq t^{*}\right\}$, where $t^{*}$ is a number (depending on $\delta$ ) such that the region is in $D^{\varepsilon}$. As $q_{x}^{\varepsilon} e^{k t}$ attains its extreme on the lateral boundary, $e^{k t} q_{x x}^{\varepsilon}\left(x_{1}^{\varepsilon}\right) \leq 0$ on the lateral boundary. Since initially $q_{0 x x}^{\varepsilon}<0$, we then conclude from the maximum principle for the equation $\left(q_{x x}^{\varepsilon}\right)_{t}=\mathbf{L} q_{x x}^{\varepsilon}$ that $q_{x x}^{\varepsilon}>0$ in the set $\left\{(x, t)|| e^{k t} q_{x}^{\varepsilon} \mid<\delta, 0 \leq t \leq t^{*}\right\}$; in particular, $p_{x x}^{\varepsilon}\left(s_{1}^{\varepsilon}(t), t\right)<0$ for all $t \in\left[0, t^{*}\right]$. Upon sending $\delta$ to 0 , we have $t^{*} \rightarrow \infty$. Hence, $p_{x x}^{\varepsilon}\left(s_{1}^{\varepsilon}(t), t\right)<0$ for all $t \geq 0$. Thus, $s_{1}^{\varepsilon}$ is smooth. 
Finally, from the differential equation $q_{t}^{\varepsilon}=\mathbf{L} q^{\varepsilon}$ and the fact that on $x=s_{1}^{\varepsilon}(t), q^{\varepsilon}>$ $0, q_{x}^{\varepsilon}=0$, and $q_{x x}^{\varepsilon}<0$, we see that $q_{t}^{\varepsilon}\left(s_{1}^{\varepsilon}(t), t\right)<0$ for all $t \geq 0$. This completes the proof.

Next we consider $q_{t}^{\varepsilon}$ in the set $D_{1}^{\varepsilon}=:\left\{(x, t) \mid x \in\left[s^{\varepsilon}(t), s_{1}^{\varepsilon}(t)\right], t \geq 0\right\}$. Differentiating $q^{\varepsilon}\left(s^{\varepsilon}(t), t\right)=0$ gives $q_{t}^{\varepsilon}=-\dot{s} q_{x}^{\varepsilon}>0$ at $x=s^{\varepsilon}(t)$. From the previous lemma, we have $q_{t}^{\varepsilon}<$ 0 when $x=s_{1}^{\varepsilon}(t)$. Hence, if we assume that the initial value $q_{t}^{\varepsilon}(x, 0)=\mathbf{L} q_{0}^{\varepsilon}(x)$ vanishes on $\left[0, x_{1}^{\varepsilon}\right]$ exactly once, we can use the same argument just given to show that $q_{t}^{\varepsilon}(\cdot, t)=0$ has exactly one root in $\left(s^{\varepsilon}(t), s_{1}^{\varepsilon}(t)\right)$ for every $t \geq 0$, as described in the following:

Lemma 3.2. Assume that $q_{0}^{\varepsilon}$ satisfies (2.3), (2.6), (3.1), and for some $x_{2}^{\varepsilon} \in$ $\left(0, x_{1}^{\varepsilon}\right), \mathbf{L} q_{0}^{\varepsilon}\left(x_{2}^{\varepsilon}\right)=0$

$$
\mathbf{L} q_{0}^{\varepsilon}(x)>0 \forall x \in\left[0, x_{2}^{\varepsilon}\right), \quad \mathbf{L} q_{0}^{\varepsilon}(x)<0 \forall x \in\left(x_{2}^{\varepsilon}, x_{1}^{\varepsilon}\right], \quad \text { and }\left(\mathbf{L} q_{0}^{\varepsilon}\right)_{x}\left(x_{2}^{\varepsilon}\right)<0 .
$$

Then there exists a smooth function $s_{2}^{\varepsilon}(t)$ defined on $[0, \infty)$ such that $s_{2}^{\varepsilon}(0)=x_{2}^{\varepsilon}$ and for all $t \geq 0$,

$$
\begin{aligned}
& s^{\varepsilon}(t)<s_{2}^{\varepsilon}(t)<s_{1}^{\varepsilon}(t), \quad q_{t}^{\varepsilon}\left(s_{2}^{\varepsilon}(t), t\right)=0, \\
& q_{t}^{\varepsilon}(x, t)>0 \forall x \in\left[s^{\varepsilon}(t), s_{2}^{\varepsilon}(t)\right), \quad q_{t}^{\varepsilon}(x, t)<0 \forall x \in\left(s_{2}^{\varepsilon}(t), s_{1}^{\varepsilon}(t)\right] .
\end{aligned}
$$

Now we are ready to consider, in the domain $\bar{D}_{2}^{\varepsilon}:=\left\{(x, t) \mid s^{\varepsilon}(t) \leq x<s_{2}^{\varepsilon}(t), t \geq 0\right\}$, the function

$$
\phi^{\varepsilon}(x, t):=\frac{q_{t}^{\varepsilon}(x, t)}{q_{x}^{\varepsilon}(x, t)} .
$$

From the two lemmas we just proved, $\phi^{\varepsilon}$ is well-defined, $\phi^{\varepsilon}(x, t)>0$ in $D_{2}^{\varepsilon}$, and $\phi^{\varepsilon}\left(s_{2}^{\varepsilon}(t), t\right)=0$.

Upon differentiating $q^{\varepsilon}\left(s^{\varepsilon}(t), t\right)=0$ we see that

$$
\dot{s}^{\varepsilon}(t)=-\phi^{\varepsilon}\left(s^{\varepsilon}(t), t\right) \text { for all } t \geq 0 .
$$

Hence, $\ddot{s}^{\varepsilon}>0$ is equivalent to $\dot{\phi}^{\varepsilon}\left(s^{\varepsilon}(t), t\right)<0$. Direct differentiation shows that in $D_{2}^{\varepsilon}$,

$$
\begin{aligned}
\phi_{t}^{\varepsilon} & =\phi_{x x}^{\varepsilon}+b^{\varepsilon} \phi_{x}^{\varepsilon}, \\
\left(\phi_{x}^{\varepsilon}\right)_{t} & =\left(\phi_{x}^{\varepsilon}\right)_{x x}+b^{\varepsilon}\left(\phi_{x}^{\varepsilon}\right)_{x}+b_{x}^{\varepsilon} \phi_{x}^{\varepsilon},
\end{aligned}
$$

where $b^{\varepsilon}=k-1+2 q_{x x}^{\varepsilon} / q_{x}^{\varepsilon}$ is a smooth function on $\bar{D}_{2}^{\varepsilon}$. This provides the PDE satisfied by $\phi_{x}^{\varepsilon}$.

Next, we consider the behavior of $\phi_{x}^{\varepsilon}$ at the boundary $x=s^{\varepsilon}(t)$. Direct differentiation gives

$$
\begin{aligned}
\phi_{x}^{\varepsilon}\left(s^{\varepsilon}(t), t\right) & =\frac{q_{t x}^{\varepsilon} q_{x}^{\varepsilon}-q_{t}^{\varepsilon} q_{x x}^{\varepsilon}}{q_{x}^{\varepsilon}}=\frac{q_{x}^{\varepsilon}\left(q_{t x}^{\varepsilon}+q_{x x}^{\varepsilon} \dot{s}^{\varepsilon}\right)-q_{x x}^{\varepsilon}\left(q_{x}^{\varepsilon} \dot{s}^{\varepsilon}+q_{t}^{\varepsilon}\right)}{q_{x}^{\varepsilon 2}} \\
& =\frac{\dot{q}_{x}^{\varepsilon}\left(s^{\varepsilon}(t), t\right)}{q_{x}^{\varepsilon}}=\frac{\ddot{s}^{\varepsilon}(t)}{\dot{s}^{\varepsilon}(t)} .
\end{aligned}
$$

As $\phi^{\varepsilon}\left(s^{\varepsilon}(t), t\right)=-\dot{s}^{\varepsilon}$, we have

$$
\dot{\phi}^{\varepsilon}\left(s^{\varepsilon}(t), t\right)=\phi^{\varepsilon} \phi_{x}^{\varepsilon}
$$


Using $\dot{\phi}^{\varepsilon}\left(s^{\varepsilon}, t\right)=\phi_{t}^{\varepsilon}+\phi_{x}^{\varepsilon} \dot{s}^{\varepsilon}=\phi_{x x}^{\varepsilon}+b^{\varepsilon} \phi_{x}^{\varepsilon}-\phi^{\varepsilon} \phi_{x}^{\varepsilon}$ we then obtain the boundary condition for $\phi_{x}^{\varepsilon}$ :

$$
\phi_{x x}^{\varepsilon}=\left(2 \phi^{\varepsilon}-b^{\varepsilon}\right) \phi_{x}^{\varepsilon} \text { on } x=s^{\varepsilon}(t), t \geq 0 .
$$

Now we are ready to prove the following.

Lemma 3.3. Assume that $q_{0}^{\varepsilon}$ satisfies (2.3), (2.6), (3.1), (3.2), and

$$
\frac{d}{d x}\left(\frac{\mathbf{L} q_{0}^{\varepsilon}}{q_{0 x}^{\varepsilon}}\right)<0 \text { on }\left[0, x_{2}^{\varepsilon}\right] .
$$

Then

$$
\ddot{s}^{\varepsilon}=-\dot{\phi}^{\varepsilon}\left(s^{\varepsilon}(\boldsymbol{t}), t\right)>0 \text { for all } t \geq 0 .
$$

Proof. We know $\phi^{\varepsilon}=q_{t}^{\varepsilon} / q_{x}^{\varepsilon}>0$ in $D_{2}^{\varepsilon}$. In view of (3.6), we need only show $\phi_{x}^{\varepsilon}<0$ in $D_{2}^{\varepsilon}$.

Initially when $t=0, \phi_{x}^{\varepsilon}=\left(\frac{q_{t}^{\varepsilon}}{q_{x}^{\varepsilon}}\right)_{x}=\left(\frac{\mathbf{L} q_{0}^{\varepsilon}}{q_{0 x}^{\varepsilon}}\right)_{x}<0$ for all $x \in\left[0, x_{2}^{\varepsilon}\right]$.

On the lateral boundary $x=s_{2}^{\varepsilon}(t)$ of the domain $D_{2}^{\varepsilon}, \phi^{\varepsilon}$ attains its minimum zero so that $\phi_{x}^{\varepsilon}\left(s_{2}^{\varepsilon}(t), t\right)<0$ for all $t \geq 0$.

Now we can conclude that $\phi_{x}^{\varepsilon}<0$ in $\bar{D}_{2}^{\varepsilon}$. Indeed, if it were not true, then there would be a minimum $t_{0}>0$ and a point $x_{0} \in\left[s^{\varepsilon}\left(t_{0}\right), s_{2}^{\varepsilon}\left(t_{0}\right)\right]$ such that $\phi_{x}^{\varepsilon}\left(x_{0}, t_{0}\right)=0$ is the maximum of $\phi_{x}^{\varepsilon}$ in $\bar{D}_{2}^{\varepsilon}\left(t_{0}\right):=\left\{(x, t) \mid t \in\left[0, t_{0}\right], x \in\left[s^{\varepsilon}(t), s_{2}^{\varepsilon}(t)\right]\right\}$. From the PDE for $\phi_{x}^{\varepsilon}$ in (3.5), we see that $\left(x_{0}, t_{0}\right)$ must lie on the lateral boundary $\bar{D}_{2}^{\varepsilon}\left(t_{0}\right)$. From the initial and boundary value of $\phi_{x}^{\varepsilon}$, we then conclude that $x_{0}=s^{\varepsilon}\left(t_{0}\right)$. By the Hopf Lemma, this implies that $\phi_{x x}^{\varepsilon}\left(x_{0}, t_{0}\right)<0$. However, from (3.7) we would have $\phi_{x x}^{\varepsilon}\left(x_{0}, t_{0}\right)=0$, and we obtain a contradiction.

Thus, $\phi_{x}^{\varepsilon}<0$ in $\bar{D}_{2}^{\varepsilon}$. Consequently, (3.9) holds. This completes the proof.

Finally we show that we can construct $q_{0}^{\varepsilon}$ having the needed properties.

LEMMA 3.4. For each small $\varepsilon>0$, there exists $q_{0}^{\varepsilon}$ satisfying (2.3) (2.6), (3.1), (3.2), and (3.8).

The construction will be given in the Appendix.

Proof of Theorem 1. Let $\left\{q_{0}^{\varepsilon}\right\}_{0<\varepsilon<\varepsilon_{0}}$ be constructed as in Lemma 3.4. Then the resulting solution $\left(q^{\varepsilon}, s^{\varepsilon}\right)$ to $(2.1)$ has the property $\dot{s}^{\varepsilon}<0$ for all $t \in[0, \infty)$. Sending $\varepsilon \searrow 0$ we see that $\ddot{s} \geq 0$.

To show that $\ddot{s}>0$, we argue as follows. Define $Q^{\varepsilon}(y, t)=q^{\varepsilon}\left(s^{\varepsilon}(t)+y, t\right)$ and $Q(y, t)=$ $q(s(t)+y, t)$. Then, together with all derivatives, $Q^{\varepsilon} \rightarrow Q$ uniformly on $[0,1] \times[\delta, 1 / \delta]$, for every $\delta>0$. It then follows that $\phi=q_{t} / q_{x}$ is non-negative and $\phi_{x}$ is non-positive near $x=s(t)$ (on the right-hand side). A strong maximum principle then gives $\ddot{s}>0$. This completes the proof.

\section{AN APPLICATION}

Now we use the convexity of $s(t)$ to find its behavior near $t=0$. Let

$$
\Gamma(x, t)=\frac{1}{\sqrt{4 \pi t}} \exp \left\{-\frac{x^{2}}{4 t}-\frac{k-1}{2} x-\frac{(k+1)^{2}}{4} t\right\}
$$


be the fundamental solution for the parabolic operator $\frac{\partial}{\partial t}-\mathbf{L}$. Applying the Green's formula for $q^{\varepsilon}$ and sending $\varepsilon \rightarrow 0$, we obtain (Chen and Chadam, 2006)

$$
\frac{\Gamma(s(t), t)}{k}=-\int_{0}^{t} \Gamma(s(t)-s(\tau), t-\tau) \dot{s}(\tau) d \tau \quad \text { for all } t>0 .
$$

To evaluate the integral, we make the change of variables from $\tau$ to $\eta$ by $\eta=\eta(t ; \tau):=$ $\frac{s(\tau)-s(t)}{2 \sqrt{t-\tau}}$. Then the interval $[0, t]$ of integration for $\tau$ becomes $[0, \alpha(t)]$ for $\eta$, where $\alpha(t)=$ $|s(t)| /(2 \sqrt{t})$. Direct differentiation gives

$$
\frac{d \eta(t ; \tau)}{d \tau}=\frac{\dot{s}(\tau)(1-\theta)}{2 \sqrt{t-\tau}}, \quad \theta=\theta(t ; \tau):=\frac{s(t)-s(\tau)}{2(t-\tau) \dot{s}(\tau)} .
$$

Using the convexity of $s$, we see that $0 \leq \theta \leq 1 / 2$. Denote by $\tau=\tau(t ; \eta)$ the inverse of $\eta=\eta(t ; \tau)$, we then obtain from (4.1) that

$$
\frac{e^{-\alpha^{2}(t)}}{\sqrt{4 \pi k^{2} t}}=\frac{1}{\sqrt{\pi}} \int_{0}^{\alpha(t)} e^{-\eta^{2}} \frac{e^{\delta(\eta)}}{1-\theta(t ; \tau(t ; \eta))} d \eta=: \mu(t)
$$

where $\delta(\eta)=(k-1) s(\tau) / 2+(k+1)^{2} \tau /\left.4\right|_{\tau=\tau(t ; \eta)}$ is a small quantity that can be ignored.

Since $\theta \in[0,1 / 2], \mu(t)$ is bounded, so we must have $\alpha(t) \rightarrow \infty$ as $t \searrow 0$. In addition, by the mean value theorem, $\mu(t) \in[1 / 2+o(1), 1+o(1)]$ where $o(1) \rightarrow 0$ as $t \searrow 0$. Thus

$$
s(t)=-2 \sqrt{t} \alpha(t), \quad \alpha(t)=\sqrt{-\log \sqrt{4 \pi k^{2} \mu^{2} t}}=\sqrt{-\log \sqrt{t}}+\frac{O(1)}{\sqrt{-\log t}} .
$$

We now show that $\mu(t) \rightarrow 1$ as $t \searrow 0$. First of all, since $s$ is convex, for any small positive $\varepsilon$,

$$
|\dot{s}(t)| \geq \frac{|s(t+\varepsilon t)-s(t)|}{\varepsilon t}=\frac{|s(t)|}{t} \frac{\sqrt{1+\varepsilon} \alpha(t+\varepsilon t)-\alpha(t)}{\varepsilon \alpha(t)}=\frac{|s(t)|}{2 t}\left\{1+O(\varepsilon)+\frac{O(1)}{\varepsilon \log t}\right\}
$$

by using (4.2). Similarly, $|\dot{s}(t)| \leq \frac{|s(t)-s(t-\varepsilon t)|}{\varepsilon t}=\frac{|s(t)|}{2 t}\left\{1+O(\varepsilon)+\frac{O(1)}{\varepsilon \log t}\right\}$. Therefore

$$
\dot{s}(t)=\frac{s(t)}{2 t}[1+o(1)]
$$

where $o(1) \rightarrow 0$ as $t \searrow 0$.

Now fix an arbitrary small positive $\varepsilon$. We write

$$
\sqrt{\pi} \mu(t)=\int_{\eta(t ; t-\varepsilon t)}^{\alpha(t)} \frac{e^{-\eta^{2}} e^{\delta(\eta)}}{1-\theta} d \eta+\int_{0}^{\eta(t ; t-\varepsilon t)} \frac{e^{-\eta^{2}} e^{\delta(\eta)}}{1-\theta(t ; \tau(t, \eta))} d \eta .
$$

When $\tau \in(0, t-\varepsilon t)$,

$$
\eta(t ; \tau) \geq \eta(t ; t-\varepsilon t)=\frac{|s(t)|}{2 t} \frac{1-s(t-\varepsilon t) / s(t)}{\sqrt{\varepsilon}} \geq \alpha(t)\left\{\sqrt{\varepsilon}-O(\varepsilon)-\frac{O(1)}{\sqrt{\varepsilon} \log t}\right\}
$$

by using (4.2). Thus $\lim _{t \searrow 0} \eta(t ; t-\varepsilon t)=\infty$. Hence, the first integral in (4.3) approaches 0 as $t \searrow 0$.

When $\tau \in[t-\varepsilon t, t], \eta \in[0, \eta(t ; t-\varepsilon t)]$ and $\theta(t ; \tau)=\frac{s(t)-s(\tau)}{(t-\tau) \dot{s}(\tau)}=1+O(\varepsilon)+\frac{O(1)}{\varepsilon \log t}$. Thus, first sending $t \searrow 0$ and then $\varepsilon \rightarrow 0$ we see that $\mu \rightarrow 1$ as $t \rightarrow 0$. Consequently,

$$
\frac{s^{2}(t)}{4 t}=\alpha^{2}(t)=-\log \sqrt{4 \pi k^{2} \mu^{2} t}=-\log \sqrt{4 \pi k^{2} t}+o(1)
$$

where $o(1) \rightarrow 0$ as $t \searrow 0$. This completes the proof of Theorem 1.2. 


\section{APPENDIX}

Proof of Lemma 2.2. For the operator $\boldsymbol{L}$, its characteristic equation $\lambda^{2}+(k-1) \lambda-$ $k=0$ has roots 1 and $-k$. Hence, the general bounded solution to $\mathbf{L} p_{0}^{\varepsilon}=-k$ for $x \in$ $(-\infty, 0]$ is given by

$$
p_{0}^{\varepsilon}(x)=1-e^{x}+c_{1} e^{x} \quad \forall x \leq 0
$$

where $c_{1}$ is any constant. By variation of constants, the general bounded solution to $\mathbf{L} p_{0}^{\varepsilon}=q_{0}^{\varepsilon}$ for $x \in[0, \infty)$ is given by

$$
p_{0}^{\varepsilon}=\frac{e^{-k x}}{1+k}\left\{c_{2}-\int_{0}^{x} q_{0}^{\varepsilon}(\xi) e^{k \xi}\right\}-\frac{e^{x}}{1+k} \int_{x}^{\infty} q_{0}^{\varepsilon}(\xi) e^{-\xi} d \xi \quad \forall x \geq 0
$$

where $c_{2}$ is an arbitrary constant.

Hence, for $p_{0}^{\varepsilon}$ to be a $C^{1}(\mathbb{R})$ bounded solution on $\mathbb{R}$, it is necessary and sufficient to take

$$
c_{2}=1, \quad c_{1}=\frac{1}{1+k}\left\{1-\int_{0}^{\infty} e^{-\xi} q_{0}^{\varepsilon}(\xi) d \xi\right\} .
$$

This proves the existence of a unique solution $p_{0}^{\varepsilon}$. The limit of $p_{0}^{\varepsilon}$ as $\varepsilon \searrow 0$ follows from the assumption (2.6) for $q_{0}^{\varepsilon}$.

Proof of Lemma 3.4. We take

$$
q_{0}^{\varepsilon}(x):=\varepsilon^{-2} Q_{\varepsilon}\left(\varepsilon^{-2} x\right), \quad Q_{\varepsilon}(y):=\left[a \varepsilon y+y^{2} / 2\right] e^{-y}
$$

where $a=a(\varepsilon, k)=\sqrt{k}+O(\varepsilon)$ is the unique positive solution to $a^{2}=k[(1-2 a \varepsilon)+(k-$ 1) $\left.a \varepsilon^{3}\right]$.

One can calculate

$$
Q_{\varepsilon}(y)=\left[a \varepsilon+(1-a \varepsilon) y-y^{2} / 2\right] e^{-y}, \quad Q_{\varepsilon}^{\prime \prime}(y)=\left[(1-2 a \varepsilon)-(2-a \varepsilon) y+y^{2} / 2\right] e^{-y} .
$$

Hence, we have the following:

(i) $q_{0}^{\varepsilon} \in C^{\infty}([0, \infty)) \cap L^{1}([0, \infty)), q_{0}^{\varepsilon}(0)=0$ and $q_{0}^{\varepsilon}(x)>0$ for all $x>0$. In addition,

$$
q_{0}^{\varepsilon}(0)^{2}-k \mathbf{L} q_{0}^{\varepsilon}(0)=\varepsilon^{-8} Q_{\varepsilon}^{\prime}(0)^{2}-k \varepsilon^{-6}\left[Q_{\varepsilon}^{\prime \prime}(0)+\varepsilon^{2}(k-1) Q_{\varepsilon}^{\prime}(0)\right\}=0
$$

by the definition of $a$. Thus, (2.3) holds.

(ii) $\int_{0}^{\infty} q_{0}^{\varepsilon}(x) d x=\int_{0}^{\infty} Q_{\varepsilon}(y) d y=1+a \varepsilon \rightarrow 1$ as $\varepsilon \searrow 0$. Also for each fixed $z>$ $0, \int_{0}^{z} q_{0}^{\varepsilon}(x) d x=\int_{0}^{z / \varepsilon^{2}} Q_{\varepsilon}(y) d y \rightarrow 1$ as $\varepsilon \searrow 0$. Hence, (2.6) holds.

(iii) Let $y_{1}^{\varepsilon}=2+O(\varepsilon)$ be the unique positive solution to $a \varepsilon+(1-a \varepsilon) y-y^{2} / 2=0$, then (3.1) holds with $x_{1}^{\varepsilon}=\varepsilon^{2} y_{1}^{\varepsilon}=2 \varepsilon^{2}+O\left(\varepsilon^{3}\right)$.

(iv) If $x \in\left[0, x_{1}^{\varepsilon}\right]$, then $y=: \varepsilon^{-2} x \in\left[0, y_{1}^{\varepsilon}\right]=[0,2+O(\varepsilon)]$ and

$$
\begin{aligned}
\mathbf{L} q_{0}^{\varepsilon}(x) & =\varepsilon^{-6}\left\{Q_{\varepsilon}^{\prime \prime}(y)+\varepsilon^{2}(k-1) Q_{\varepsilon}^{\prime}(y)-k \varepsilon^{4} Q_{\varepsilon}(y)\right\} \\
& =\varepsilon^{-6} e^{-y}\left\{1-2 y+y^{2} / 2+O(\varepsilon)\right\} .
\end{aligned}
$$

It follows that there exists $y_{2}^{\varepsilon}=2-\sqrt{2}+O(\varepsilon)$ such that (3.2) holds with $x_{2}^{\varepsilon}=$ $\varepsilon^{2} y_{2}^{\varepsilon}$. 
(v) Finally, for $x \in\left[0, x_{2}^{\varepsilon}\right]$, we have $y=\varepsilon^{-2} x \in[0,2-\sqrt{2}+O(\varepsilon)]$ and

$$
\begin{aligned}
\frac{d}{d x} \frac{\mathbf{L} q_{0}^{\varepsilon}(x)}{q_{0 x}^{\varepsilon}(x)} & =\varepsilon^{-4} \frac{d}{d y} \frac{1-2 y+y^{2} / 2+O(\varepsilon)}{a \varepsilon+(1-a \varepsilon) y-y^{2} / 2} \\
& =\varepsilon^{-4} \frac{O(\varepsilon)-[2-y]\left[1-y^{2} / 2\right]-\left[1-2 y+y^{2} / 2\right][1-y]}{\left[a \varepsilon+(1-a \varepsilon) y-y^{2} / 2\right]^{2}}<0 .
\end{aligned}
$$

Thus (3.8) holds. This completes the proof.

\section{REFERENCES}

Aitsahlia, F., and T. LAi (2001): Exercise Boundaries and Efficient Approximations to American Option Prices and the Hedge Parameters, J. Computational Finance 7, 85-103.

Barone-Adesi, G., and R. E. Whaley (1987): Efficient Analytic Approximation of American Option Values, J. Financ. 42, 301-320.

BArone-Adesi, G., and R. Elliott (1991): Approximations for the Values of American Options, Stochast. Anal. Appl. 9, 115-131.

Barles, G., J. Burdeau, M. Romano, and N. Samsoen (1995): Critical Stock Price Near Expiration, Math. Financ. 5, 77-95.

Broadie, M., and J. Detemple (1996): American Option Valuation: New Bounds, Approximations and Comparison of Existing Methods, Rev. Finan. Stud. 9, 1121-1250.

Bunch, D. A., and H. Johnson (2000): The American Put Option and Its Critical Stock Price, J. Financ. 55, 2333-2356.

CARr, P., R. JARrow, and R. Myneni (1992): Alternative Characterization of American Put Option, Math. Financ. 2, 87-105.

Chen Xinfu, and J. ChadAm (2006): A Mathematical Analysis of the Optimal Exercise Boundary American Put Options, SIAM J. Mathematical Analysis 38, 1613-1641.

Chen, X., and J. CHADAm (2003): Analytic and Numerical Approximations for the Early Exercise Boundary for American Put Options, Dyn. Cont. Disc. Impulsive Sys. 10, 649-657.

Ekstrom, E. (2004): Convexity of the Optimal Stopping Boundary for the American Put Option, J. Math. Anal. Appl. 299, 147-156.

Friedman, A. (1982): Variational Principles and Free Boundary Problems, New York: John Wiley $\&$ Sons.

Friedman, A. (1976): Analyticity of the Free Boundary for the Stefan Problem, Arch. Rational Mech. Anal. 61, 97-125.

Friedman, A., and R. Jensen (1977): Convexity of Free Boundary in the Stefan Problem and in the Dam Problem, Arch. Rational Mech. Anal. 67, 1-24.

Hull, J. (1997): Options, Futures and Other Derivative Securities, 3rd Ed., New York: PrenticeHall.

JIANG, L. (1965): Existence and Differentiability of the Solution of a Two Phase Stefan Problem for Quasi-Linear Parabolic Equations, Chinese Math. Acta 7, 481-496.

KIM, I. J. (1990): The Analytic Evaluation of American Options, Rev. Finan. Stud. 3, 547-572.

Kuske, R. A., and J. B. Keller (1998): Optimal Exercise Boundary for an American Put Option, Appl. Math. Financ. 5, 107-116.

MacMillan, L. W. (1986): Analytic Approximation for the American Put Option, Adv. Future Opt. Res. 1 (A), 119-139. 
McKean, H. P. Jr. (1965): Appendix: A Free Boundary Problem for the Heat Equation Arising from a Problem in Mathematical Economics, Ind. Manage. Rev. 6, 32-39.

Merton, R. (1992): Continuous-Time Finance, Oxford: Blackwell.

VAn Moerbeke, P. (1976): On Optimal Stopping and Free Boundary Problems, Arch. Rational Mech. Anal. 60, 101-148.

Stamicar, R., D., Ševčovič, and J. Chadam (1999): The Early Exercise Boundary for the American Put Near Expiry: Numerical Approximation, Canad. Appl. Math. Quart. 7, 427444.

Wilmott, P., J. Dewynne, and S. Howison (1995): Option Princing: Mathematical Models and Computation, New York: Cambridge University Press. 